\title{
Expression and immune recognition of Brugia malayi VAL-1, a homologue of vespid venom allergens and Ancylostoma secreted proteins $^{\text {is }}$
}

\author{
Janice Murray a, William F. Gregory a, Natalia Gomez-Escobar a, \\ Agnes Kurniawan Atmadja ${ }^{b}$, Rick M. Maizels ${ }^{\mathrm{a}, *}$ \\ a Ashworth Laboratories, Institute of Cell, Animal and Population Biology, University of Edinburgh, West Mains Road, \\ Edinburgh EH9 3JT, UK \\ ${ }^{\mathrm{b}}$ Department of Parasitology, University of Indonesia, Jakarta, Indonesia
}

Received 18 January 2001; received in revised form 27 August 2001; accepted 27 August 2001

\begin{abstract}
Several important nematode parasites have been found to express members of a gene family variously termed as venom allergen antigen homologue (vah) or Ancylostoma secreted protein (asp). In some cases these products are secreted by infective larval stages and have been suggested to be effective vaccine immunogens. We isolated the corresponding gene from the human filarial nematode, Brugia malayi, by first searching the expressed sequence tag (EST) dataset generated by the Filarial Genome Project and then using gene-specific nondegenerate primers matching the selected gene for PCR, from B. malayi cDNA libraries. We report here the full-length gene sequence, which we have designated as Bm-val-1, for vah/asp-like. The corresponding protein (Bm-VAL-1) contains 232 amino acids in a single homology unit, unlike products from some other species in which there is a tandem repeat. A putative signal sequence is present at the $5^{\prime}$ end and there are two potential $N$-glycosylation sites. Murine antibodies to recombinant Bm-VAL-1 react with a $28 \mathrm{kDa}$ protein in L3 extracts and recombinant Bm-VAL-1 is recognised by murine T cells primed with soluble L3 proteins. Of 82 ESTs corresponding to Bm-val-1, 72 are recorded from the infective larval (L3) stage. However, PCR on the first-strand cDNA libraries from later mammalian stages revealed some expression at most subsequent time points. Over 95\% (20/21) of microfilaraemic human filariasis patients are seropositive for antibodies to $B m$-VAL-1, with particularly high levels of IgG3 and IgG4 isotypes. The IgG4 subclass may indicate stimulation by adult and/or microfilarial-derived immunogens. The association of Bm-VAL-1 with the infective stage and its recognition by humans exposed to filariasis suggests that further evaluation of this antigen as a vaccine candidate should be performed. C) 2001 Published by Elsevier Science B.V.
\end{abstract}

Keywords: Filariasis; Nematodes; Infective larvae; Isotypes; Vaccines

\section{Introduction}

The infective third-stage larvae of many parasitic nematodes undergo the most critical transition of the life cycle, from a free-living or arthropod-borne existence to the definitive, often mammalian, host [1,2].

\footnotetext{
Note: Nucleotide sequence data reported in this paper have been submitted to the GenBank ${ }^{\mathrm{TM}}$ data base with accession number AF334661.

* Corresponding author. Tel.: + 44-131-650-5511; fax: + 44-131650-5450.

E-mail address: rick.maizels@ed.ac.uk (R.M. Maizels).
}

Products from these stages are of particular interest as key mediators of the invasion process and as potential immunogens for anti-parasite vaccines [3-5]. When similar proteins are associated with larval stages of many different nematode species, a common pathway or essential component of the infection process may be inferred. One such instance is the group of proteins first described as Ancylostoma caninum secreted protein or ASP [6] with similarity to a wider family including, for example, hymenopteran venom allergens. Significantly, these products are released when resting larvae are stimulated to commence invasion. The venom allergen antigen homologue VAH/ASP family contains two dis- 
tinct forms, typified in $A$. caninum [7]: $A c$-ASP-1 is a long-form $42 \mathrm{kDa}$ protein while $A c$-ASP-2 is a shortform $20-22 \mathrm{kDa}$ product. Sequence analysis reveals that $A c$-ASP-1 contains a tandem nonidentical repeat of an ancestral unit represented only once in $A c$-ASP-2 [7].

Members of this gene family have now been reported from a range of nematode species. In the sheep intestinal nematode Haemonchus contortus, both the largeform Hc40 [8] and short-form Hc24 [9,10] have been identified. Multiple homologues have been discovered in the two human hookworm species Ancylostoma duodenale and Necator americanus [11,12], and also in Meloidogyne incognita [13], Onchocerca volvulus [14] and Toxocara canis [15]. Additional gene members in Ascaris and Strongyloides have also been indicated by hybridisation experiments with Ac-asp-2 probes [7]. Further, a homologue from the filarial nematode Dirofilaria immitis was selected by reactivity with immune dog serum (Tripp C, Wisnewski N, unpublished deposition) and a related gene in Brugia malayi reported from expressed sequence tag (EST) analysis [4]. Significantly, up to 17 homologous genes have been identified in the genome of Caenorhabditis elegans $[7,12]$. All but one of these are single-unit forms (e.g. $C$. elegans C39E9.1), the exception being F11C7.3 which displays a paired but nonidentical repeat structure [8].

More distant relatives of the VAH/ASP family can be found across a wide evolutionary spectrum. Within A. caninum, a related gene encodes a neutrophil inhibitory factor (NIF), which displays an antagonistic binding to the integrin receptor $\alpha_{M} \beta_{2}(\mathrm{CD} 11 \mathrm{~b} / \mathrm{CD} 18$, Mac-1) on mammalian cells [16]. While the aforementioned insect venom allergens have no known function, they are similar to helothermine, a lizard salivary toxin, which blocks the ryanodine receptor [17]. In mammals, similar proteins have been described as the testis-specific proteins TPX-1, sperm-coating glycoprotein (SCG) and cysteine-rich secretory protein (CRISP) [18], as well as P25TI, a novel trypsin inhibitory protein upregulated in some tumour lines [19]. The larger gene family also includes an interesting set of plant defence proteins, all characterised by a conserved signature of key residues [7]. From these similarities, it is difficult to deduce a general function for nematode homologues, but it is attractive to postulate inhibition or activation of host receptor-dependent processes. For example, the $O v$ ASP-1 and $O v$-ASP-2 proteins from Onchocerca volvulus have been shown to promote corneal angiogenesis when injected into mice [14], a role in keeping with the formation of vascularised nodules in onchocerciasis.

Because vaccines against nematode parasites focus on the infective larval stage, heightened expression of vah/ asp genes in larvae make these products an attractive candidate vaccine antigen [20]. Promising results have so far been obtained in two different species. Thus, four out of five sheep vaccinated with a fraction enriched with $H$. contortus Hc-24 were protected against establishment of adult parasites [21], while ASP-1-immunised mice show up to $80 \%$ reduction in infection $[22,23]$.

The Filarial Genome Project has provided a major resource and impetus for gene discovery from $B$. malayi and other filarial nematodes [4,24-28]. EST sequences deposited for $B$. malayi include a number corresponding to a homologue of ASP [4,25,28]. We describe here the characterisation of this homologue, and its expression as a recombinant probe for immunological reactivity of patients infected with this major human tropical pathogen.

\section{Materials and methods}

\subsection{Database searching}

The ungapped TBLASTN algorithm was used with the $A$. caninum ASP-1 protein sequence matched against all deposits in the NCBI dbEST nucleotide database translated into all six frames [29]. Multiple ESTs with significant similarity were found, permitting a putative full-length sequence to be assembled. From this, a $5^{\prime}$ gene-specific primer was designed to amplify the gene by PCR from an L3 cDNA library, 5'-AGT ACT TAC TAG ACG ACA TCT TAC TGT T-3' (roman typeface nt -68 to $-54,5^{\prime}$ of the start codon), in conjunction with M13 reverse primer for a $3^{\prime}$ vector sequence. This product was cloned and sequenced in full.

\subsection{PCR amplifications and $5^{\prime} R A C E$}

The $5^{\prime}$ end of the Bm-val-1 cDNA was amplified using the rapid amplification of cDNA ends (RACE) procedure following the supplier's instructions (GibcoBRL). Starting with mixed adult B. malayi total RNA, first-strand cDNA was synthesised from a primer sequence close to the $5^{\prime}$ end of the cDNA clone; this primer was 5'-TAG TCG ACC TCC TGG ACA TTC$3^{\prime}$ (complementary to nt $72-52$ of cDNA in which the initiating ATG codon is numbered 1-3). PCR was then performed with an anchor primer supplied in the kit and a second gene specific primer: 5'-AAC GAA AAA TAA CAT TTT GCC GAG-3' (complementary to nt 15 to -9 ). The PCR products were ligated into the pMOS-blue vector. Clones were picked at random and fully sequenced.

\subsection{Reverse transcription- $P C R$}

First-strand cDNA was produced from total RNA using GeneAmp RT-PCR kits (Applied Biosystems, Cheshire, UK) as previously described [5]. For PCR, 
val-1- specific primers were used on first-strand cDNA for 30 cycles of $1 \mathrm{~min}$ at $99{ }^{\circ} \mathrm{C}, 1 \mathrm{~min}$ at $55{ }^{\circ} \mathrm{C}$ and 1.5 min at $72{ }^{\circ} \mathrm{C}$. The primers used were $A$ TT TGA ATG TCC AGG AGG TCG A, corresponding to nt 49-69, and TTT TTC TAC ACA ATC CAG ATT T, corresponding to nt 660-640; in each case an additional nucleotide (italicised) was added at the $5^{\prime}$ end.

\subsection{Expression and antibody analysis}

Recombinant $B m$-VAL-1 protein was expressed in $E$. coli by cloning the presumed mature coding sequence (a 609 bp insert encoding aa 17-220) into the pET-29T vector (Novagen), following PCR with the two primers described above (49-69 and 660-640). Protein expression was induced in BL21(DE3) cells with $1 \mathrm{mM}$ IPTG for $3 \mathrm{~h}$ at $37{ }^{\circ} \mathrm{C}$. Bacteria were pelleted and sonicated in a $6 \mathrm{M}$ urea buffer suitable for metal chelating chromatography on His-bind resin (Novagen). Eluted protein was dialysed against PBS, and recovered at a final concentration of $2 \mathrm{mg} \mathrm{ml}^{-1}$. Both $\mathrm{BALB} / \mathrm{c}$ and $\mathrm{CBA} / \mathrm{Ca}$ mouse strains were immunised with two doses of $10 \mu \mathrm{g} \mathrm{rBm}$-VAL-1 (first in complete Freund's adjuvant, the second in incomplete), one month apart. Serum samples taken 7 and 14 days following the boost showed that only the BALB/c responded with a polyclonal antibody response. For Western blots, whole microfilarial (Mf), L3 and adult worms were solublised directly in PBS, or $1.5 \% n$-octylglucoside ( $n \mathrm{OG}$, Sigma), or in SDS-PAGE loading buffer. Samples were subjected to SDS-PAGE and electrotransfer, then probed with a $1 / 200$ dilution of murine anti- $B m$-VAL-1 or normal mouse serum, followed by peroxidase antimouse Ig (BioRad, 1/2000) and ECL substrate.

\subsection{Murine $T$ cell reactivity}

$\mathrm{BALB} / \mathrm{c}$ mice were immunised with $10 \mu \mathrm{g}$ of soluble extract of L3 mixed with an equal volume of complete Freund's adjuvant (Sigma) in the rear footpad. Ten days later, popliteal lymph node cells were recovered and cultured as described in detail elsewhere [30]. The data were compared by the two-tailed $t$-test and a $P$ value of 0.05 or less was considered significant.

\subsection{ELISA}

Sera from 42 adult individuals residing in the Palu Valley area of Central Sulawesi, Indonesia, which is endemic for B. malayi filariasis, were tested for isotype specific antibodies to recombinant $B m$-VAL-1. Of these patients, 21 were patent microfilaraemic (range 40$3300 \mathrm{Mf} \mathrm{ml}^{-1}$ ) and 21 asymptomatic amicrofilaraemic (exposed but 'endemic normal') individuals. Recombinant antigen was coated onto Nunc immunoplates at 1 $\mu \mathrm{g} \mathrm{ml}^{-1}$ in $0.06 \mathrm{M}$ carbonate buffer and plates were otherwise treated as described previously for analyses of responses to whole B. malayi antigens $[31,32]$.

\section{Results and discussion}

\subsection{Search of dbEST for ASP homologues}

The A. caninum ASP-1 sequence was used to screen the NCBI dbEST database containing the Filarial Genome Project EST sequences from multiple stages of B. malayi. Of approximately 18700 sequences, 82 showed high levels of similarity and 72 of these were from the infective L3 stage larval cDNA library. This represents an abundance of $2.22 \%$ (72/3249 L3 ESTs). Similar levels of abundance have been observed for the related nematode $O$. volvulus [14]. Three more cDNAs were noted from the Mf stage, five from post-infection larvae and two from adult male worms [28]. Closer inspection of these sequences indicated that all were derived from the same gene, which has been assigned a single cluster number (BMC00351) by the Filarial Genome Project.

\subsection{PCR amplification and $5^{\prime} R A C E$}

The longest EST contained an open reading frame of 220 amino acids, equivalent to a single homology unit of $A c$-ASP-1. As Ac-ASP-1 and H. contortus Hc40 were known to contain tandemly duplicated domains, the possibility that Bm-val-1 encodes a larger protein was investigated. From first-strand cDNA of mixed adult-stage parasites, a primer close to the known $5^{\prime}$ end was used for $5^{\prime}$ RACE. However, no longer product was obtained in the two experiments, although the same protocol and reagents were successful for other transcripts [33]. No product was obtained in amplifications with the nematode 22-nt spliced leader and a $B m-v a l-1$ specific primer, although parallel conditions amplified a number of other larval cDNAs [2]. All ESTs for which appropriate sequence had been recorded encoded a stop codon at nt 661-663 indicating that no second $3^{\prime}$ domain exists.

\subsection{Sequence of B. malayi vespid (venom allergen)/Ancylostoma (secreted protein)-like product $(B m-V A L-1)$}

The open reading frame encodes 220 amino acids, of which the first 16 represent a putative signal sequence. The presence of this hydrophobic motif further indicates that the sequence represented here is the authentic full-length product. This sequence differs at two nucleotide positions from that deposited by McCarthy and Hopkins (AF042088). These substitutions are each synonymous and occur at nt 219 and 351. Of 17 ESTs for 
which data are available, $11(65 \%)$ have the two nucleotides reported by McCarthy and six (35\%) have the two we describe here. Such differences may well represent allelic forms among the B. malayi gene pool, as noted at other loci [34]. This indication of sequence polymorphisms is not surprising in the light of Qiang et al.'s recent report of no less than 42 nucleotide variations (of which 12 were nonsynonymous) among $A c$ asp-1 genes from different A. caninum isolates [35]. Other features of the protein sequence include two $\mathrm{N}$-glycosylation sites and a preponderance of cysteine residues in the C-terminal segment (aa 164-220), which contains seven of the ten cysteines in the protein.

\subsection{Evolutionary relationship of VAH/ASP-like (VAL) family members}

The nematode VAL proteins show characteristics of a larger protein family, designated pfam00188 and also known as SCP (sperm coating protein)-like with homologues of mammalian, insect, fungal and plant origins. The same family has also been named CAP, to denote Cysteine-rich secretory proteins of mammals, vespid venom Allergens, and plant Pathogenesis-related proteins [36]. Bm-VAL-1 shares 17 of the 19 residues identified as conserved across the whole CAP family (Fig. 1).
Cladograms summarising the likely phylogeny of these genes have recently been published in which all the filarial VAL sequences form a monophyletic group distinct from other nematode genes, with $97-100 \%$ bootstrap support $[12,14]$. Within the filarial nematodes, $B m$-VAL-1 is most similar to $W b$-VAH-1, sharing 91\% amino acid identity (Fig. 1). Other filarial VAL sequences in the public databases include one from the dog heartworm (D. immitis) and three homologues from $O$. volvulus, of which only $O v$-ASP-3 is expressed by the L3 stage [14]. ESTs for a VAL homologue are also available for Litomosoides sigmodontis [37]. The complete filarial sequences, together with the canonical A. caninum ASP and a representative venom allergen from the hymenopteran fire ant, are also compared in Fig. 1.

\subsection{Expression by different life cycle stages}

The pattern of expression of Bm-val-1 can be followed through the mammalian life cycle of $B$. malay $i$ by RT-PCR from first-strand cDNAs taken at 1- to 2-day intervals over the first 4 weeks' infection of jirds from L3 to the adult stage. The mRNA is strongly represented in the mosquito-borne L3 stage, as well as at certain other life cycle points such as maturing L4 and young adult worms (Fig. 2). Moreover, expression occurs in the Mf stage as reflected in ESTs derived from

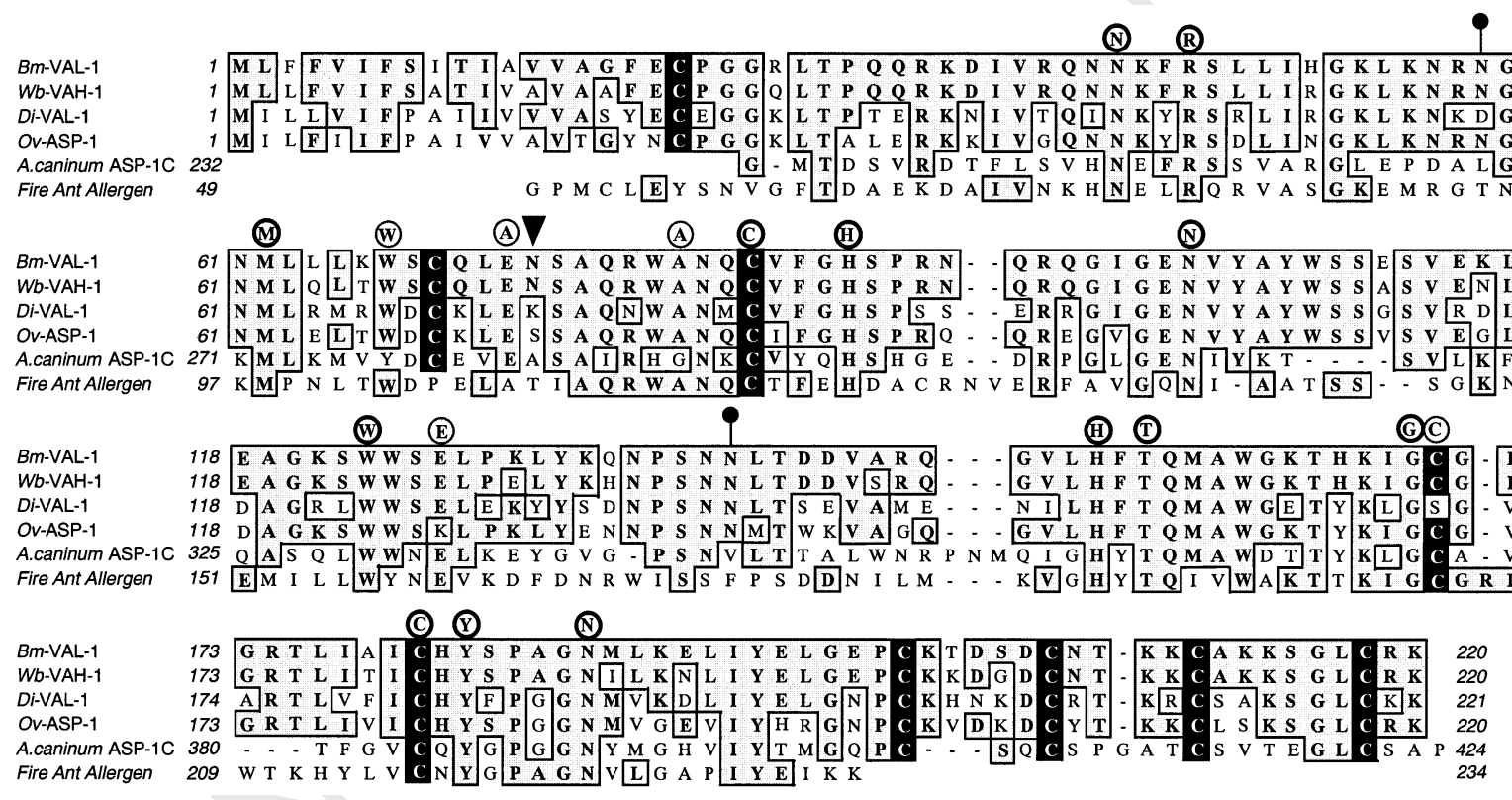

Fig. 1. Alignment of filarial VAL proteins with homologues from Ancylostoma and the fire ant. Authors' designations of genes as VAH or ASP have been respected. Alignments were prepared using the ClustalW facility on MacVector 7.0. All cysteine residues are displayed in black. Lollipop symbols identify the two potential $N$-glycosylation sites, both of which are shared with $W b$-VAH-1, and one of which is present in $D i$-VAL-1. Inverted triangles denote codons found to show synonymous nucleotide polymorphisms (nt 217/219, AAC/AAT, both Asn; and nt 349/351 ACC/ACG, both Thr). Residues identified as characteristic of the CAP family from mammals, insects, nematodes and plants [35] are circled above the alignment, heavier shading denoting conservation within the nematode sequences shown. Accession numbers of the sequences presented are as follows: $W$. bancrofti VAH-1, AF109794; Dirofilaria immitis VAL, AF001100; O. volvulus ASP-1, AF020586; A. caninum ASP-1, AF132291; Solonepsis invicta (fire ant) allergen sol i 3, AF012919. 


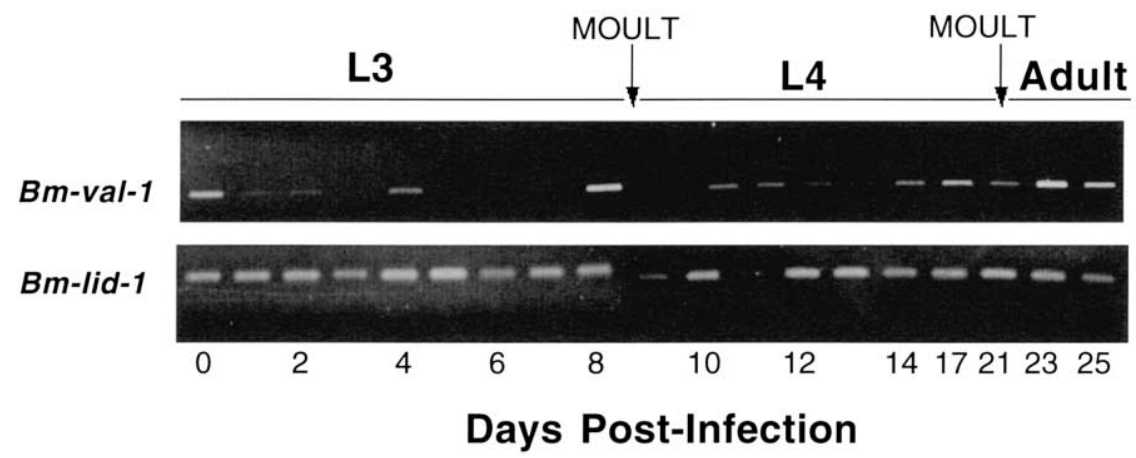

Fig. 2. Expression of Bm-val-1 during the mammalian stages of the B. malayi life cycle. The stage and timing of the moults following infection of the mammalian host are indicated above the panel showing PCR products amplified from each time point. d0 represents mosquito-derived L3. mRNA taken at each time point was used to make first-strand cDNA as templates in PCR reactions using the Bm-val-1-specific primer pair detailed in Section 2. As a control, primers for Bm-lid-1 (a homologue of Ov-L3 from O. volvulus, Murray J, Maizels RM, unpublished) show constant expression from all stages tested.

the Mf library. Thus, transcription does not show complete developmental restriction.

\subsection{Native Bm-VAL-1 is a $28 \mathrm{kDa}$ L3 protein}

Expression of the Bm-VAL-1 protein was also monitored using a polyclonal antibody raised to the recombinant protein. Western blot analysis with PBS-soluble extracts of the three major life cycle stages (Mf, L3 and adults) reveals antibody-reactive proteins in both $\mathrm{Mf}$ and L3 (Fig. 3A). The L3 component has an estimated molecular weight (mol. wt.) of $28 \mathrm{kDa}$. A strong $\mathrm{Mf}$ band can also be seen with a slightly faster mobility, but no corresponding antigen is present in adult extracts. Interestingly, some fainter bands are apparent in the higher mol. wt. ranges. Since many nematode species are known to encode multiple VAL-type genes (e.g. two in Haemonchus, $\geq 9$ in Necator, 17 in C. elegans), it is possible that these weaker reactions represent related but distinct VAL gene products.

While $B m$-VAL-1 protein is present in PBS-soluble extracts, some of the product requires detergent for its release (Fig. 3B). Moreover, the harsher conditions of SDS-2ME solubilise a reactive form of approximately $40 \mathrm{kDa}$. Again, comparison with other species suggests that this may represent a different member of the same gene family in B. malayi.

Several experiments were undertaken to establish if $B m$-VAL-1 is secreted by infective larvae. Overnight incubation of mosquito-derived L3 in a variety of conditions (1000 L3 ml-1 of RPMI or Grace's medium at 26 or $37^{\circ} \mathrm{C}$ ) did not result in the release of sufficient $B m$-VAL-1 protein to be detected by Western blot, although another highly-expressed larval protein $(\mathrm{Bm}$ ALT-1) could readily be observed under these conditions. If Brugia larvae, like Ancylostoma larvae, require a developmental cue to stimulate release of the ASPlike VAL protein, it could be argued that only exposure to mammalian factors in vivo can provide the necessary signal. Thus, the question of whether Bm-VAL-1 functions as an exo-antigen within the host environment remains to be resolved.

\subsection{Expression and reactivity of $B m-V A L-1$}

A panel of human filariasis sera was tested for antibody recognition of recombinant $B m$-VAL-1-protein and high levels of serological reactivity were observed (Fig. 4). Two groups of individuals were studied: asymptomatic amicrofilaraemic persons, who are clinically normal but exposed and may harbour cryptic infections $(\mathrm{Mf}-)$; and microfilaraemic patients with circulating parasites $(\mathrm{Mf}+)$. In the first subset of endemic normals, $18 / 21(86 \%)$ were seropositive in at least one of the $4 \mathrm{IgG}$ subclasses of antibodies to $\mathrm{Bm}$-VAL1 , while in $\mathrm{Mf}+$ patients, the proportion was $20 / 21$ $(95 \%)$. The most vigorous antibody isotypes observed were $\mathrm{IgG} 3$ and $\mathrm{IgG} 4$.

The IgG3 isotype has previously been linked to protective immunity to the L3 stage [38-40], while the presence of IgG4 is strongly associated with exposure to adult worms and microfilariae [41]. Because previous work suggests that larval antigens do not elicit IgG4 responses [32], the presence of anti-Bm-VAL-1 IgG4 may reflect a low level of expression by adult and microfilarial stages.

\subsection{Immunogenicity to $T$ cell populations}

To test whether $\mathrm{T}$ cell responses were likely to be mounted to VAL, mice were immunised with soluble proteins from the infective larval stage. When primed lymph node cells were challenged in vitro with $\mathrm{rBm}$ VAL-1, a significant proliferative response was observed in every experiment (Fig. 5; $P<0.005$ ). Assays for antigen-specific cytokine release showed that levels of IL-4 and IFN- $\gamma$ produced in response to Bm-VAL-1 were only marginally above background (no-antigen 
control) values (data not shown). Human $\mathrm{T}$ cell responses were also gauged by stimulation of PBMC from filariasis patients. Positive proliferative responses were observed in some patients, but in only one case was significant production of IFN- $\gamma$ noted (Sartono E, Yazdanbakhsh M, personal communication).

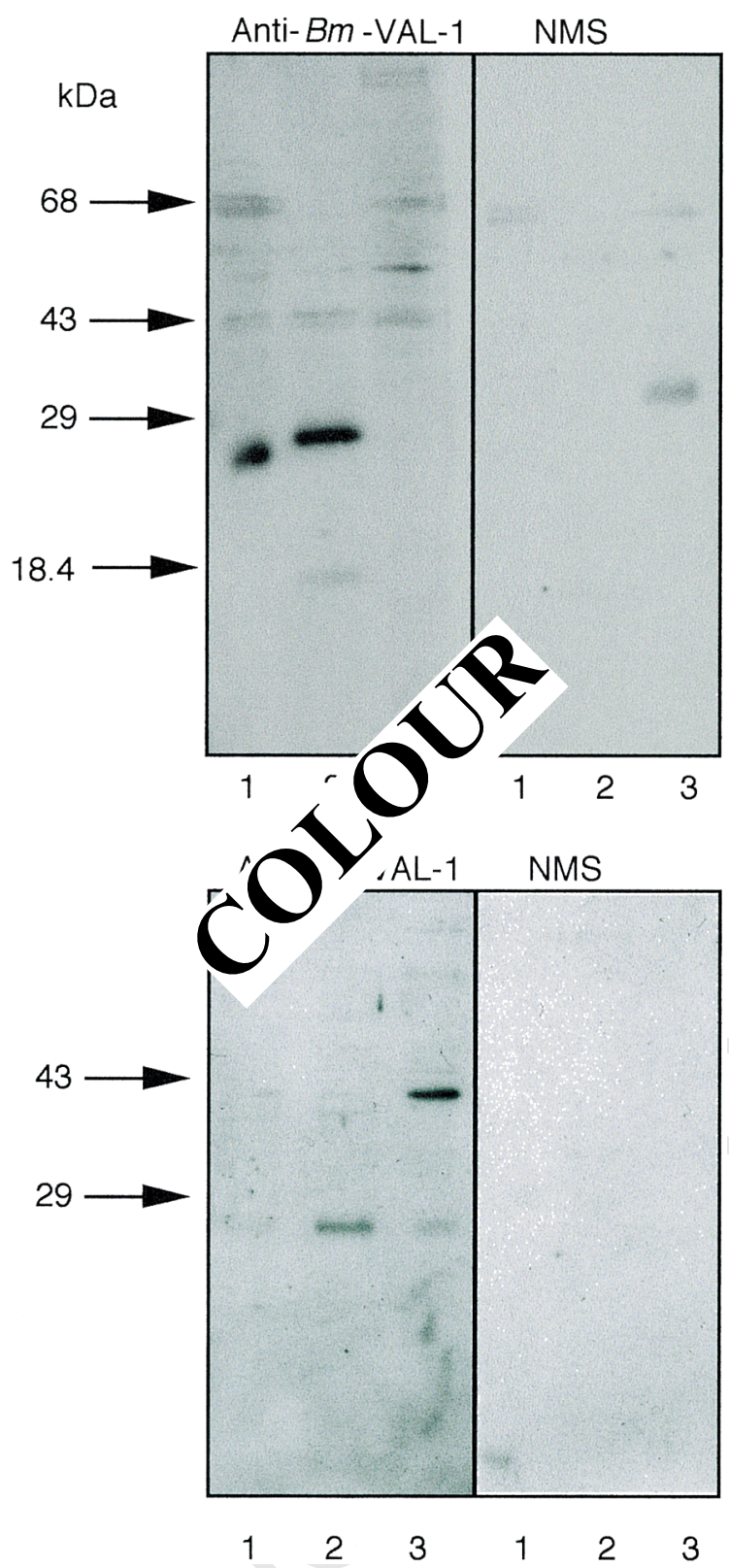

Fig. 3. Western Blot identification of native Bm-VAL-1 antigens. (A) Mf (track 1) and L3 (track 2) $10 \mu \mathrm{g}$ of PBS-soluble extracts were probed with polyclonal mouse antibody to recombinant $B m$-VAL-1 (left panel). No reaction was observed with adult extracts (track 3) or with normal mouse serum (NMS, right panel). (B) L3 extracted sequentially with PBS (track 1), nOG (track 2) and SDS-PAGE loading buffer (track 3 ) were probed with mouse antibody to $\mathrm{r} B m$ VAL-1 (left panel). No reaction was observed with NMS (right panel).

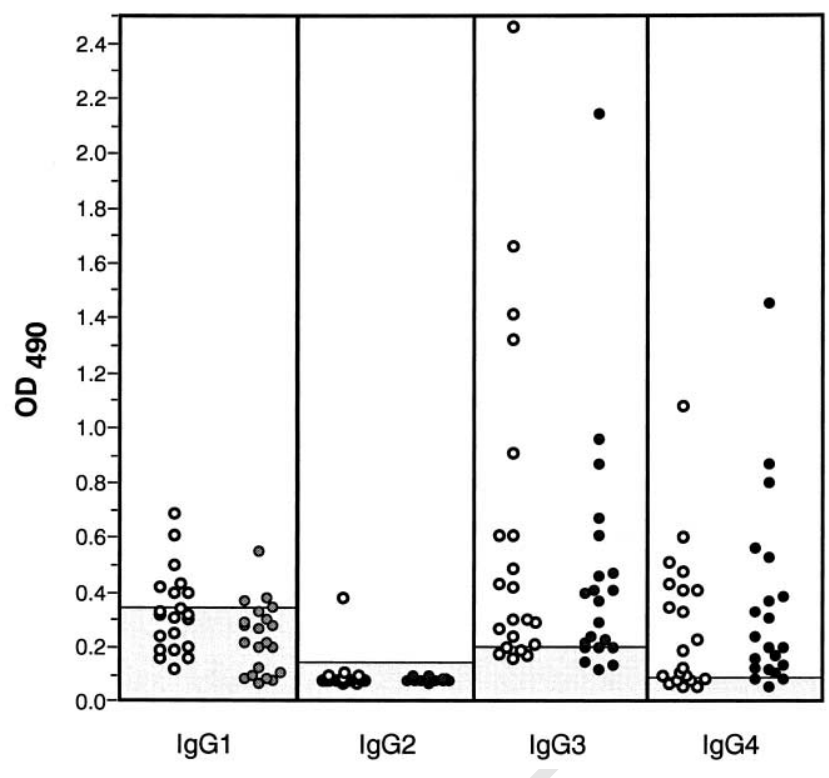

Fig. 4. Specific antibody responses in human filariasis patients. Antibody levels specific for Bm-VAL-1 in the sera of 21 Mf-negative endemic normal subjects $(\bullet)$ and 21 microfilaraemic patients $(\bigcirc)$ were measured by ELISA for $\mathrm{IgG} 1, \mathrm{IgG} 2, \mathrm{IgG} 3$ and $\mathrm{IgG} 4$ using plates coated with $1 \mu \mathrm{g} \mathrm{ml}^{-1}$ recombinant antigen. All patients were adults residing in an area of Central Sulawesi, Indonesia, endemic for B. malayi. The cut-off values are represented by the shaded boxes and are derived from the mean of three European normal control values plus three SD.

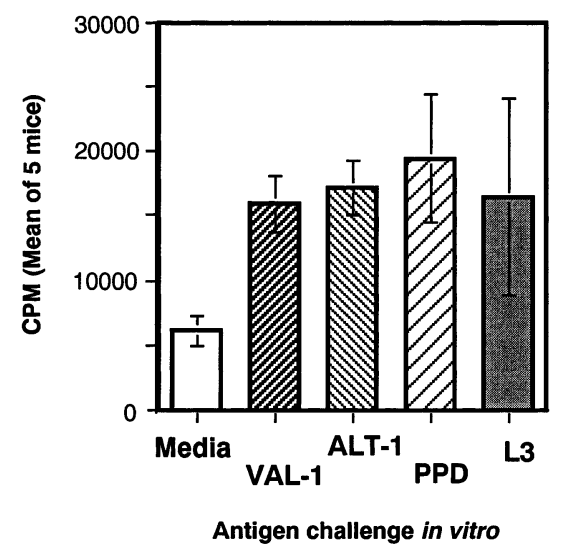

Fig. 5. T cells from L3-immunised mice respond to Bm-VAL-1. $\mathrm{BALB} / \mathrm{c}$ mice were immunised with $10 \mu \mathrm{g}$ of soluble L3 extract proteins in complete Freund's adjuvant subcutaneously in the footpad. Ten days later, popliteal lymph node cells were taken and cultured in vitro in the presence of $10 \mu \mathrm{g} \mathrm{ml}^{-1}$ recombinant VAL protein. Positive controls included Bm-ALT-1 (an abundant L3 protein [5]), mycobacterial PPD (a constituent of complete Freund's adjuvant) and the L3 extract used for initial immunisation. Data represent the means and standard deviations from groups of five mice. The response to $B m$-VAL-1 was significantly above mediaalone controls by a two-tailed $t$-test $(P<0.005)$. 


\subsection{Vaccination}

Because of the prominence of Bm-val-1 among larval cDNA transcripts and the success of similar products in vaccination against other nematode species, recombinant Bm-VAL-1 was used to immunise jirds. Following four injections (the first of $75 \mu \mathrm{g}$ in complete Freund's adjuvant, two boosts of $25 \mu \mathrm{g}$ in incomplete Freund's adjuvant and a final boost of $7.5 \mu \mathrm{g}$ in IFA), jirds were challenged with 300 mosquito-derived infective larvae intraperitoneally. Four weeks later, the Bm-VAL-1-immunised group showed a mean reduction of $64 \%$ in parasite recovery from the peritoneal cavity (immunised: geometric mean 18.6, range 7-108; control given adjuvant alone: geometric mean 50.9, range 13-195). Although a substantial difference, this failed to reach statistical significance (Mann-Whitney nonparametric test, group sizes $=6$ ). A similar experiment was then conducted, but again, no significant effect was observed and only minimal reductions in recovery obtained. Future work may aim to refine the immunisation protocol and to combine Bm-VAL-1 with other candidate immunogens in a multivalent vaccine.

Vaccination against filarial nematodes offers a robust strategy for eradication of this major tropical scourge [42-44]. Recently, the recombinant B. malayi larvalspecific antigen Bm-ALT-1 was reported to elicit protective responses in jirds [5]. The expression of $B m$-VAL-1 is less stringently restricted to the L3 than is the case for Bm-ALT-1 and the possibility remains that VAL immunisation could prime pathogenic responses against mature stages. Future vaccination studies will need also to assess whether vigorous immune responses to nematode VAL proteins carry any risk of harmful reactivities either to environmental antigens (such as may be encountered from insect bites), or to the endogenous proteins in mammalian hosts such as the testis-specific autoantigen Tpx [45].

\section{Conclusions}

The VAL family has become one of the most intensively studied sets of genes from nematode parasites, showing strong association with larval invasion of the mammalian host. We report here that these products do not go unnoticed by the host immune system and suggest that an appropriate response to these antigens may prove to be protective. It will now be important to establish the biological function of the VAL proteins in the host-parasite interaction, so that we can begin to appreciate a fuller picture of how VAL products may promote parasite infection and how immune responses to these antigens may frustrate parasite survival.

\section{Acknowledgements}

We thank Mark Blaxter for providing an analysis of the frequency of ESTs corresponding to the val-1 gene and for the constructive comments on the manuscript. We are also grateful for Yvonne Harcus, Judith Allen and Laetitia Le Goff for their invaluable assistance with the vaccination trials. The studies described here were funded by the Wellcome Trust and the European Union (INCO-DC1C18-CT95-0014 and INCODC1C18-CT95-0245).

\section{References}

[1] Bianco AE, Robertson BD, Kuo Y-M, Townson S, Ham P. Developmentally regulated expression and secretion of a polymorphic antigen by Onchocerca infective-stage larvae. Mol Biochem Parasitol 1990;39:203-12.

[2] Gregory WF, Blaxter ML, Maizels RM. Differentially expressed, abundant trans-spliced cDNAs from larval Brugia malayi. Mol Biochem Parasitol 1997;87:85-95.

[3] Ibrahim MS, Richie TL, Scott AL. Surface-associated antigens of Brugia malayi L2 and L3 parasites during vector-stage development. Mol Biochem Parasitol 1992;52:97-110.

[4] Blaxter ML, Raghavan N, Ghosh I, et al. Genes expressed in Brugia malayi infective third stage larvae. Mol Biochem Parasitol 1996;77:77-93.

[5] Gregory WF, Atmadja AK, Allen JE, Maizels RM. The abundant larval transcript $1 / 2$ genes of Brugia malayi encode stagespecific candidate vaccine antigens for filariasis. Infect Immun 2000;68:4174-9

[6] Hawdon JM, Jones BF, Hoffman DR, Hotez PJ. Cloning and characterization of Ancylostoma-secreted protein. A novel protein associated with the transition to parasitism by infective hookworm larvae. J Biol Chem 1996;271:6672-8.

[7] Hawdon JM, Narasimhan S, Hotez PJ. Ancylostoma secreted protein 2: cloning and characterization of a second member of a family of nematode secreted proteins from Ancylostoma caninum. Mol Biochem Parasitol 1999;99:149-65.

[8] Rehman A, Jasmer DP. A tissue specific approach for analysis of membrane and secreted protein antigens from Haemonchus contortus gut and its application to diverse nematode species. Mol Biochem Parasitol 1998;97:55-68.

[9] Sharp PJ, Wagland BM, Coburn G. Haemonchus contortus vaccine. US Patent 5,525,508, 1996.

[10] Schallig HDFH, van Leeuwen MAW, Verstrepen BE, Cornelissen AWCA. Molecular characterization and expression of two putative protective excretory secretory proteins of Haemonchus contortus. Mol Biochem Parasitol 1997;88:203-13.

[11] Bin Z, Hawdon J, Qiang S, et al. Ancylostoma secreted protein 1 (ASP-1) homologues in human hookworms. Mol Biochem Parasitol 1999;98:143-9.

[12] Daub J, Loukas A, Pritchard D, Blaxter ML. A survey of genes expressed in adults of the human hookworm Necator americanus. Parasitology 2000;120:171-84.

[13] Ding X, Shields J, Allen R, Hussey RS. Molecular cloning and characterisation of a venom allergen AG5-like cDNA from Meloidogyne incognita. Int J Parasitol 2000;30:77-81.

[14] Tawe W, Pearlman E, Unnasch TR, Lustigman S. Angiogenic activity of Onchocerca volvulus recombinant proteins similar to vespid venom antigen 5. Mol Biochem Parasitol 2000;109:91-9.

[15] Tetteh KKA, Loukas A, Tripp C, Maizels RM. Identification of abundantly-expressed novel and conserved genes from infective 
stage larvae of Toxocara canis by an expressed sequence tag strategy. Infect Immun 1999;67:4771-9.

[16] Moyle M, Foster DL, McGrath DE, et al. A hookworm glycoprotein that inhibits neutrophil function is a ligand of the integrin CD11b/CD18. J Biol Chem 1994;269:10008-15.

[17] Morrissette J, Krätzschmar J, Haendler B, et al. Primary structure and properties of helothermine, a peptide toxin that blocks ryanodine receptors. Biophys J 1995;68:2280-8.

[18] Krätzschmar J, Haendler B, Eberspaecher U, Roosterman D, Donner P, Schleuning W-D. The human cysteine-rich secretory protein (CRISP) family. Primary structure and tissue distribution of CRISP-1, CRISP-2 and CRISP-3. Eur J Biochem 1996;236:827-36.

[19] Yamakawa T, Miyata S, Ogawa N, et al. cDNA cloning of a novel trypsin inhibitor with similarity to pathogenesis-related proteins, and its frequent expression in human brain cancer cells. Biochim Biophys Acta 1998;1395:202-8.

[20] Hotez PJ, Hawdon JM, Cappello M, et al. Molecular approaches to vaccinating against hookworm disease. Pediatr Res 1996;40:515-21.

[21] Schallig HDFH, van Leeuwen MAW. Protective immunity to the blood-feeding nematode Haemonchus contortus induced by vaccination with parasite low molecular weight antigens. Parasitology 1997;114:293-9.

[22] Ghosh K, Hawdon J, Hotez P. Vaccination with alum-precipitated recombinant Ancylostoma-secreted protein 1 protects mice against challenge infections with infective hookworm (Ancylostoma caninum) larvae. J Infect Dis 1996;174:1380-3.

[23] Sen L, Ghosh K, Bin Z, et al. Hookworm burden reductions in $\mathrm{BALB} / \mathrm{c}$ mice vaccinated with recombinant Ancylostoma secreted proteins (ASPs) from Ancylostoma duodenale, Ancylostoma caninum and Necator americanus. Vaccine 2000;18:1096-102.

[24] Blaxter M. The filarial genome network. Parasitol Today 1995;11:441-3.

[25] The Filarial Genome Project. Deep within the filarial genome: an update on progress in the Filarial Genome Project. Parasitol Today 1999;15:219-24.

[26] Williams SA, Lizotte-Waniewski MR, Foster J, et al. The filarial genome project: analysis of the nuclear, mitochondrial and endosymbiont genomes of Brugia malayi. Int $\mathbf{J}$ Parasitol 2000;30:411-9.

[27] Maizels RM, Gomez-Escobar N, Gregory WF, Murray J, Zang $\mathrm{X}$. Immune evasion genes from filarial nematodes. Int $\mathbf{J}$ Parasitol 2001;31:889-98.

[28] Maizels RM, Blaxter ML, Scott AL. Immunogenomics of filariasis: genes implicated in immune evasion and protective immunity. Parasite Immunol 2001;23:327-44.

[29] Altschul SF, Gish W, Miller W, Myers EW, Lipman DJ. Basic local alignment search tool. J Mol Biol 1990;215:403-10.

[30] Zang XX, Atmadja AK, Gray P, et al. The serpin secreted by Brugia malayi microfilariae, Bm-SPN-2, elicits strong, but shortlived, immune responses in mice and humans. J Immunol
2000;165:5161-9.

[31] Kurniawan A, Yazdanbakhsh M, van Ree R, et al. Differential expression of $\mathrm{IgE}$ and $\mathrm{IgG} 4$ specific antibody responses in asymptomatic and chronic human filariasis. $\mathbf{J}$ Immunol 1993; 150:3941-50.

[32] Kurniawan A, Sartono E, Partono F, Yazdanbakhsh M, Maizels RM. Antibody responses to filarial infective larvae are not dominated by the IgG4 isotype. Parasite Immunol 1998;20:9-17.

[33] Gomez-Escobar N, Lewis E, Maizels RM. A novel member of the transforming growth factor- $\beta$ (TGF- $\beta$ ) superfamily from the filarial nematodes Brugia malayi and B. pahangi. Exp Parasitol 1998;88:200-9.

[34] Gregory WF, Murray J, Abrahamson M, Maizels RM. Two cystatin-type cysteine protease inhibitors from the human filarial nematode Brugia malayi show differential developmental expression, distinct inhibition profiles, and differ in the presence or absence of a highly conserved glycine residue. Submitted for publication.

[35] Qiang S, Bin Z, Shu-hua X, Zheng F, Hotez P, Hawdon JM. Variation between ASP-1 molecules from Ancylostoma caninum in China and the United States. J Parasitol 2000;86:181-5.

[36] Megraw T, Kaufman TC, Kovalick GE. Sequence and expression of Drosophila Antigen 5-related 2, a new member of the CAP gene family. Gene 1998;222:297-304.

[37] Allen JE, Daub J, Guilliano D, et al. Analysis of genes expressed at the infective larval stage validate the utility of Litomosoides sigmodontis as a murine model for filarial vaccine development. Infect Immun 2000;68:5454-8.

[38] Day KP, Gregory WF, Maizels RM. Age-specific acquisition of immunity to infective larvae in a Bancroftian filariasis endemic area of Papua New Guinea. Parasite Immunol 1991;13:277-90.

[39] Ottesen EA. Infection and disease in lymphatic filariasis: an immunological perspective. Parasitology 1992;104:S71-9.

[40] Maizels RM, Sartono E, Kurniawan A, Selkirk ME, Partono F, Yazdanbakhsh M. T cell activation and the balance of antibody isotypes in human filariasis. Parasitol Today 1995;11:50-6.

[41] Kwan-Lim G-E, Forsyth KP, Maizels RM. Filarial-specific IgG4 response correlates with active Wuchereria bancrofti infection. J Immunol 1990;145:4298-305.

[42] Yates JA, Higashi GI. Brugia malayi: vaccination of jirds with ${ }^{60}$ Cobalt-attenuated infective stage larvae protects against homologous challenge. Am J Trop Med Hyg 1985;34:1132-7.

[43] Philipp M, Davis TB, Storey N, Carlow CKS. Immunity in filariasis: perspectives for vaccine development. Annu Rev Microbiol 1988;42:685-716

[44] Maizels RM, Allen JE, Yazdanbakhsh M. Immunology of lymphatic filariasis: current controversies. In: Nutman TB, Pasvol G, editors. Lymphatic filariasis. 2000. p. 217-43.

[45] Foster JA, Gerton GL. Autoantigen 1 of the guinea pig sperm acrosome is the homologue of mouse Tpx-1 and human TPX1 and is a member of the cysteine-rich secretory protein (CRISP) family. Mol Reprod Dev 1996;44:221-9. 